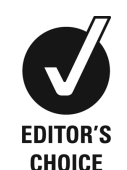

${ }^{1}$ Department of Paediatric Dentistry, MES Dental College, Perinthalmanna, Kerala, India ${ }^{2}$ Department of Oral Medicine and Radiology, MES Dental College, Perinthalmanna, Kerala, India

${ }^{3}$ Department of Paediatrics, MES Medical College, Perinthalmanna, Kerala, India

\section{Correspondence to} Professor Abdul Hafiz, hafizzzdr@gmail.com

Accepted 5 July 2015

\title{
An unusual case of KBG syndrome with unique oral findings
}

\author{
Abdul Hafiz, ${ }^{1}$ Abdulla Mufeed, ${ }^{2}$ Mohamad Ismael, ${ }^{3}$ Mheboob Alam ${ }^{3}$
}

\section{SUMMARY}

KBG syndrome is a condition characterised by macrodontia, neurological disturbance, short stature, a distinct cranio-facial appearance, and skeletal anomalies. The authors describe what appears to be the first case of KBG syndrome reported from the Indian subcontinent. Meticulous evaluation of the dental findings helps to identify such cases which may otherwise remain undiagnosed. Further research is warranted to determine the classic and variant presentations of this condition, with follow-up data providing valuable insights into its natural history and long-term prognosis.

\section{BACKGROUND}

KBG syndrome is a rare condition with four cardinal manifestations: typical facial dysmorphism, macrodontia of the maxillary central incisors, skeletal (mainly costovertebral) anomalies and developmental delay. ${ }^{1}$ However, this combination of features is observed in many genetic conditions. In addition, since many of the presenting features of KBG syndrome are mild and usually not associated with severe medical complications, it is likely to be undiagnosed. Therefore, a thorough understanding of the clinical features will help in the diagnosis and management of this condition.

\section{CASE PRESENTATION}

A 12-year-old girl was referred from a private dental clinic to the department of paediatric dentistry with complaints of a mobile primary molar. She appeared short in stature for her age and had developmental delay. She was the only child of a nonconsanguineous couple. The mother showed mild mental retardation and had an artificial prosthesis on her maxillary anterior teeth. The child's maternal grandfather, who accompanied them, was the informer.

The antenatal and postnatal periods were uneventful. The child was born full term through normal delivery. She exhibited mild developmental delay, had learning difficulties and gave a negative history of seizure disorders. A dysmorphic face, high arched eyebrows and bulbous nose were striking facial features (figure 1). She had a high palatal vault and unusually large and flared maxillary incisors (figure 2). All incisors were abnormal and were shovel-shaped with accentuated mamelons and deeply scooped lingual fossa resulting in carious lesions (figures 2-4). The molars were larger than usual and peculiar in shape. The lower molars were destroyed by caries. Most of the patient's teeth were rotated and irregularly placed

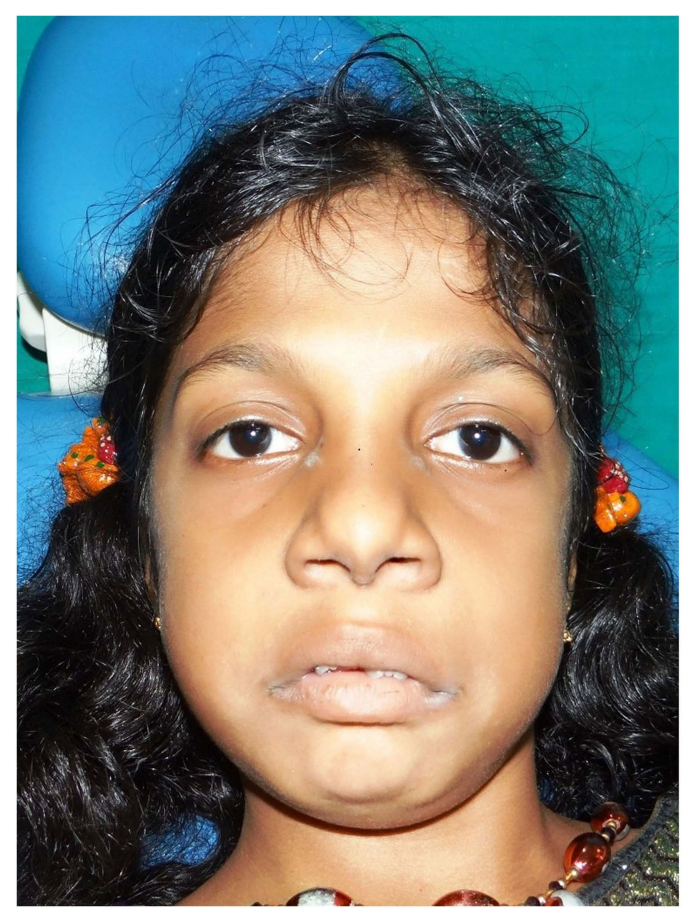

Figure 1 Dysmorphic face, high arched eyebrows and bulbous nose.

in the arch. She was evaluated by a team of paediatricians and a geneticist for further diagnostic work-up.

\section{INVESTIGATIONS}

The panoramic radiograph revealed the presence of all tooth buds but with atypical morphology. Radiographically, most of the teeth were very large

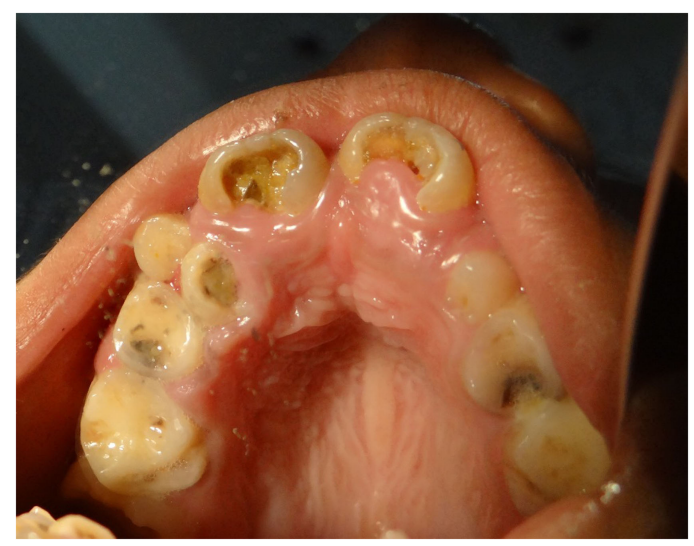

Figure 2 High arched palate, shovel-shaped tooth crowns. 


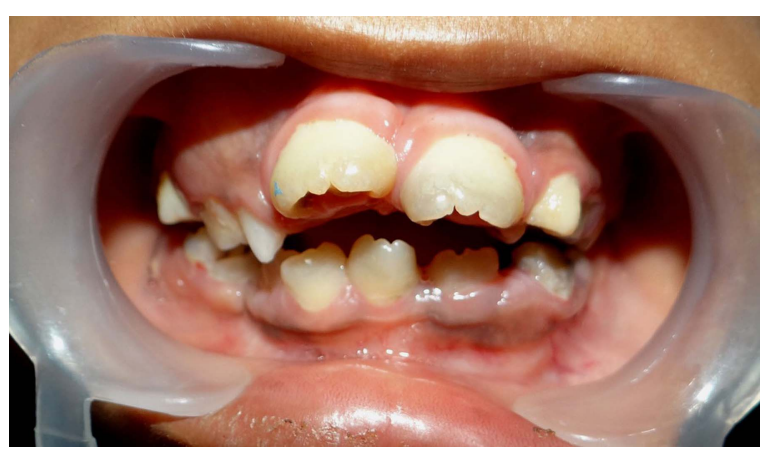

Figure 3 Macrodontic incisors with accentuated mamelons.

(macrodontia) and appeared strange (figure 5). The incisors demonstrated an irregular facet-like depression on the crowns and most of the teeth had marked constriction of the cervical region. The premolars were missing in the mandibular left quadrant and all other premolars remained unerupted. Bilateral clinodactyly was evident on hand-wrist radiographs (figure 6). Radiographic evaluation of the spine, pelvis and long bones did not reveal any other skeletal abnormalities. An EEG was

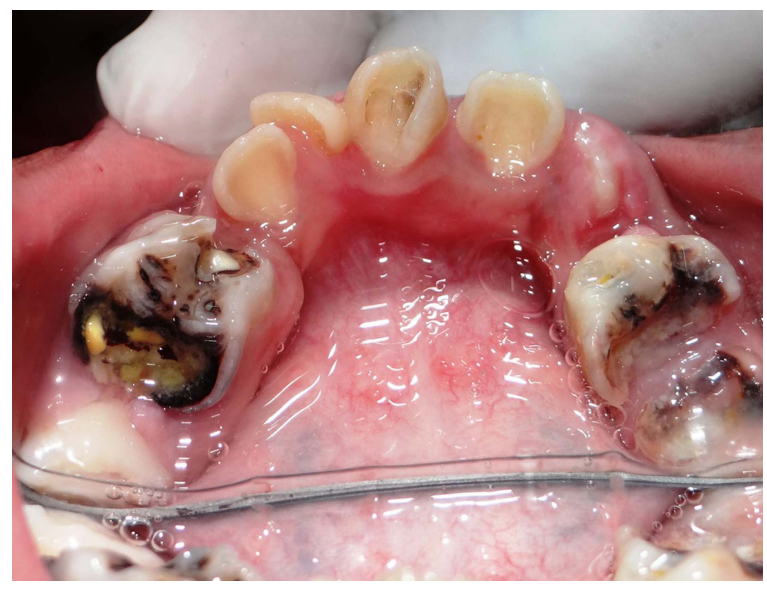

Figure 4 Mandibular anterior teeth with deeply scooped lingual fossae.

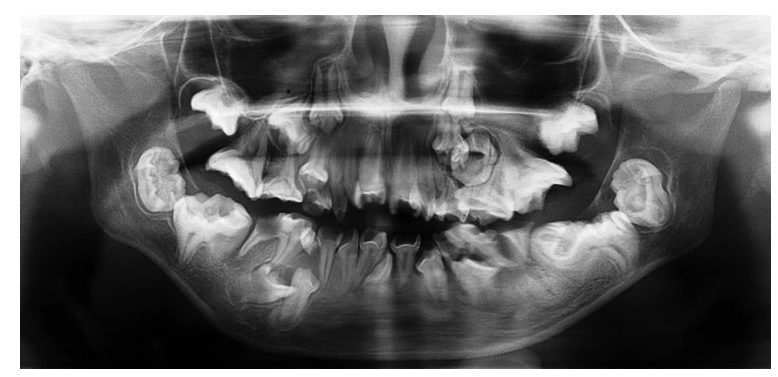

Figure 5 Orthopantogram showing generalised macrodontia and unusual tooth morphology.

performed as the patient had developmental delay and mental retardation, but showed no abnormalities.

\section{TREATMENT}

The grossly carious teeth were extracted under local anaesthetic, the accentuated lingual fossae and carious lesions were restored with glass ionomer cement, and the patient was advised to follow meticulous oral hygiene. Currently, the patient and her mother are under the supervision and care of the social rehabilitation team.

\section{OUTCOME AND FOLLOW-UP}

The patient is being regularly followed up for assessment of oral hygiene and evaluation of any carious lesions. The parents are currently undergoing genetic counselling.

\section{DISCUSSION}

KBG syndrome, first described by Herrmann and co-workers in 1975 , is a rare autosomal dominant condition. ${ }^{1}$ The letters ' $\mathrm{K}$ ', ' $\mathrm{B}$ ' and ' $\mathrm{G}$ ' stand for the initials of the three family surnames of the affected individuals documented in that report. The fundamental findings are mild to moderate developmental delay, shortness of stature, facial dysmorphism, macrodontia of the permanent maxillary incisors and skeletal anomalies. However, a large number of additional findings have also been described. ${ }^{2}$ To date, only 60 cases of KBG syndrome worldwide have been reported in the literature.

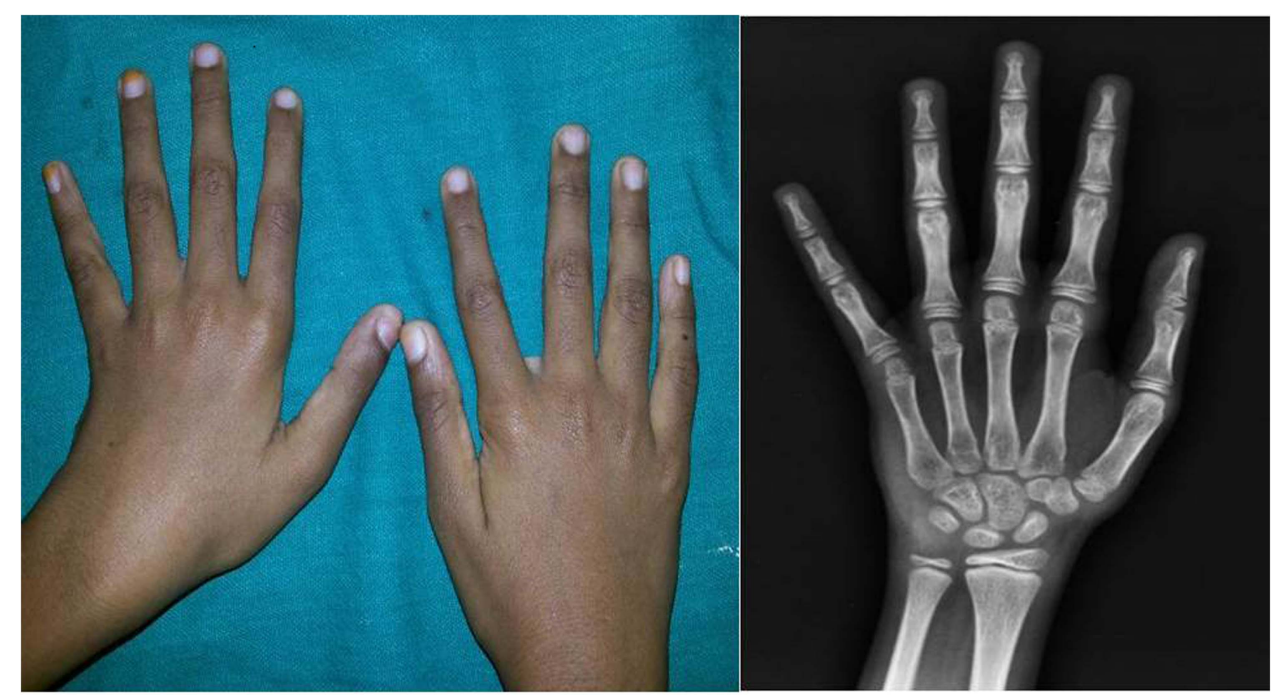

Figure 6 Clinical and radiographic evidence of clinodactyly. 
The diagnosis of KBG syndrome is usually based on the clinical findings. Diagnosis is difficult before eruption of the permanent maxillary central incisors. However, once these erupt, the characteristic appearance of the macrodontia in association with short stature and developmental delay facilitates the diagnosis. ${ }^{3}{ }^{4} \mathrm{An}$ accurate diagnosis is important for genetic counselling. ${ }^{5}$

The pattern of inheritance was thought to be autosomal dominant with variable expressivity; X-linked inheritance was also suggested in some families. ${ }^{4}$ Recently, the ANKRD11 gene was identified as being responsible for the condition. In addition, microdeletion of $16 \mathrm{q} 24.3$, including ANKRD11, has been reported to result in the KBG syndrome phenotype. ${ }^{6}$

Owing to associated cranio-facial dysmorphism, patients affected by KBG syndrome can present many challenges to the anaesthesiologist pre-operatively evaluating the management of difficult airways. ${ }^{2}$ The various clinical manifestations have been documented in the literature as major and minor features (box 1).

The combination of findings described in the present case are sufficient for a diagnosis of KBG syndrome, as they satisfy six of the suggested eight major criteria: macrodontia, characteristic

\section{Box 1 Clinical features of KBG syndrome}

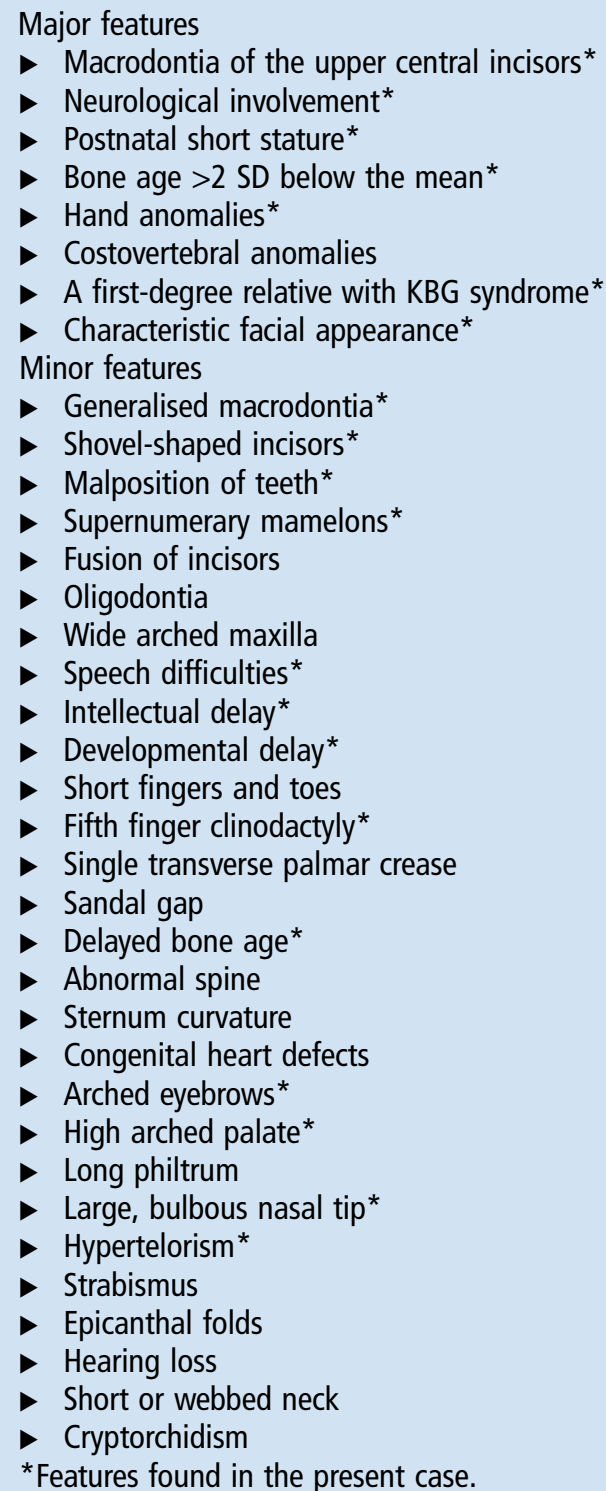

facial anomalies, hand anomalies, neurological anomalies, reduced bone age and postnatal short stature. However, the syndrome is likely to be undiagnosed as many of the presenting features are mild and usually not associated with significant medical complications. The diagnosis is seldom made before the eruption of the permanent maxillary central incisors. Macrodontia of these teeth is the single most consistent feature exhibited by the majority of patients presenting with KBG syndrome in previous reports. ${ }^{4}$ However, the unusual shovel appearance of incisors found in this case have rarely been described in previous reports. A paediatric dentist familiar with these dental anomalies can play a key role in identifying rare cases like KBG syndrome as the dental manifestations confirm the diagnosis. Furthermore, a specialist multidisciplinary team is essential for deciding on management strategies to minimise future complications. ${ }^{3}$ Caution is required while performing endodontic treatment on macrodont teeth as they tend to have enlarged pulp chambers. Care is also needed during extraction and root canal treatment as the dilated apical third of 'taurodontic' teeth can cause complications. ${ }^{7}$

Recent studies have reported skeletal improvement following growth hormone administration in patients with KBG syndrome. ${ }^{8}$

\section{Learning points}

- Orofacial findings and dental anomalies are important diagnostic features of KBG syndrome.

- Identification of rare syndromes requires an inter-disciplinary team.

- A paediatric dentist and/or oral medicine specialist can best evaluate the orofacio-dental findings for diagnosis of rare syndromes.

- Accurate diagnosis of syndromes is important for genetic counselling.

Contributors $\mathrm{AH}$ and $\mathrm{AM}$ evaluated the patient, diagnosed the condition, and carried out the dental treatment. MI and MA performed the clinical and skeletal evaluation and confirmed the diagnosis. All authors were involved in collecting data, searching the literature and final approval of the article.

Competing interests None declared.

Patient consent Obtained.

Provenance and peer review Not commissioned; externally peer reviewed.

\section{REFERENCES}

1 Herrmann J, Pallister PD, Tiddy W, et al. The KBG syndrome: a syndrome of short stature, characteristic facies, mental retardation, macrodontia and skeletal anomalies. Birth Defects Orig Artic Ser 1975;11:7-18.

2 Morghen I, Ferri E. The KBG syndrome: case report. Cases J 2008;1:186.

3 Almandey $\mathrm{AH}$, Anthonappa RP, King NM, et al. KBG syndrome: clinical features and specific dental findings. Pediatr Dent 2008;32:439-44.

4 Kumar H, Prabhu N, Cameron A. KBG syndrome: review of the literature and findings of 5 affected patients. Oral Surg Oral Med Oral Pathol Oral Radiol Endod 2009;108: e72-9.

5 Dowling PA, Fleming P, Gorlin RJ, et al. The KBG syndrome, characteristic dental findings: a case report. Intl J Paediatr Dent 2001;11:131-4.

6 Sirmaci A, Spiliopoulos M, Brancati F, et al. Mutations in ANKRD11 cause KBG syndrome, characterized by intellectual disability, skeletal malformations, and macrodontia. Am J Hum Genet 2011;89:289-94.

7 Jafarzadeh $\mathrm{H}$, Azarpazhooh A, Mayhlli JT. Taurodontism: a review of the condition and endodontic treatment challenges. Intl Endod J 2008;42:375-88.

8 Reynaert N, Ockeloen CW, Sävendahl L, et al. Short stature in KBG syndrome: first responses to growth hormone treatment. Horm Res Paediatr 2015;83:361-4. 
Copyright 2015 BMJ Publishing Group. All rights reserved. For permission to reuse any of this content visit http://group.bmj.com/group/rights-licensing/permissions.

BMJ Case Report Fellows may re-use this article for personal use and teaching without any further permission.

Become a Fellow of BMJ Case Reports today and you can:

- Submit as many cases as you like

- Enjoy fast sympathetic peer review and rapid publication of accepted articles

- Access all the published articles

- Re-use any of the published material for personal use and teaching without further permission

For information on Institutional Fellowships contact consortiasales@bmjgroup.com

Visit casereports.bmj.com for more articles like this and to become a Fellow 